\title{
Resource Theory of Quantum States Out of Thermal Equilibrium
}

\author{
Fernando G. S. L. Brandão, ${ }^{1,2}$ Michał Horodecki, ${ }^{3,4}$ Jonathan Oppenheim, ${ }^{5}$ \\ Joseph M. Renes, ${ }^{6,7, *}$ and Robert W. Spekkens ${ }^{8}$ \\ ${ }^{1}$ Departamento de Física, Universidade Federal de Minas Gerais, Caixa Postal 702, Belo Horizonte, Minas Gerais 30123-970, Brazil \\ ${ }^{2}$ Centre for Quantum Technologies, National University of Singapore, 3 Science Drive 2, Singapore 117543, Singapore \\ ${ }^{3}$ Institute for Theoretical Physics and Astrophysics, University of Gdańsk, PL-80952, Gdańsk, Poland \\ ${ }^{4}$ National Quantum Information Centre of Gdańsk, 81-824 Sopot, Poland \\ ${ }^{5}$ Department of Applied Mathematics and Theoretical Physics, University of Cambridge, Wilberforce Road, \\ Cambridge CB3 OWA, United Kingdom \\ ${ }^{6}$ Institut für Angewandte Physik, Technische Universität Darmstadt, Hochschulstrasse 4a, 64289 Darmstadt, Germany \\ ${ }^{7}$ Institut für Theoretische Physik, ETH Zurich, CH-8093 Zürich, Switzerland \\ ${ }^{8}$ Perimeter Institute for Theoretical Physics, Waterloo, Ontario N2L 2Y5, Canada
}

(Received 17 August 2012; revised manuscript received 12 March 2013; published 18 December 2013)

\begin{abstract}
The ideas of thermodynamics have proved fruitful in the setting of quantum information theory, in particular the notion that when the allowed transformations of a system are restricted, certain states of the system become useful resources with which one can prepare previously inaccessible states. The theory of entanglement is perhaps the best-known and most well-understood resource theory in this sense. Here, we return to the basic questions of thermodynamics using the formalism of resource theories developed in quantum information theory and show that the free energy of thermodynamics emerges naturally from the resource theory of energy-preserving transformations. Specifically, the free energy quantifies the amount of useful work which can be extracted from asymptotically many copies of a quantum system when using only reversible energy-preserving transformations and a thermal bath at fixed temperature. The free energy also quantifies the rate at which resource states can be reversibly interconverted asymptotically, provided that a sublinear amount of coherent superposition over energy levels is available, a situation analogous to the sublinear amount of classical communication required for entanglement dilution.
\end{abstract}

PACS numbers: 05.30. $-\mathrm{d}$, 03.67.Bg, 03.67.Mn, 05.70.-a

Quantum resource theories are specified by a restriction on the quantum operations (state preparations, measurements, and transformations) that can be implemented by one or more parties. This singles out a set of states which can be prepared under the restricted operations. If the parties facing the restriction acquire a quantum state outside the restricted set of states, then they can use this state to implement measurements and transformations that are outside the class of allowed operations, consuming the state in the process. Thus, such states are useful resources.

A few prominent examples serve to illustrate the idea: if two or more parties are restricted to communicating classically and implementing local quantum operations, then entangled states become a resource [1]; if a party is restricted to quantum operations that have a particular symmetry, then states that break this symmetry become a resource [2-4]; if a party is restricted to preparing states that are completely mixed and performing unitary operations, then any state that is not completely mixed, i.e., any state that has some purity, becomes a resource [5].

In this Letter, we develop the quantum resource theory of states that are $T$ athermal, i.e., not thermal at temperature $T$. This provides a useful new formulation of equilibrium and nonequilibrium thermodynamics for finite-dimensional quantum systems, and allows us to apply new mathematical tools to the subject. The restricted class of operations which defines our resource theory includes only those that can be achieved through energy-conserving unitaries and the preparation of any ancillary system in a thermal state at temperature $T$, as first studied by Janzing et al. [6] in the context of Landauer's principle. Here, the ancillary systems can have an arbitrary Hilbert space and an arbitrary Hamiltonian, and may be described as having access to a single heat bath at temperature $T$. States that are not in thermal equilibrium at temperature $T$ are the resource in this approach.

Quantum resource theories provide answers to questions such as: How does one measure the quality of different resource states? Can one particular resource state be converted to another deterministically? If not, can it be done nondeterministically, and if so with what probability? What if one has access to a catalyst? A particularly fundamental problem, addressed in this Letter, is to identify the equivalence classes of states that are reversibly interconvertible in the limit of asymptotically many copies of the resource and to determine the rates of interconversion. We show that all $T$-athermal states are reversibly interconvertible asymptotically and that the interconversion rate is governed by the free energies of the states involved.

The great merit of the resource theory approach is its generality. Rather than considering the behavior of the property of interest for some particular system with 
particular dynamics (as is typical in thermodynamics), one considers instead the fundamental limits that are imposed by the restriction defining the resource and the laws of quantum theory. On the practical side, a better understanding of a given resource helps determine how best to implement the tasks that make use of it, and, more fundamentally, such an understanding may serve to clarify what sorts of resources are even relevant for a given task. For instance, entanglement is commonly asserted to be the necessary resource for tasks in which the use of quantum systems yields improved performance over the use of classical systems. But in quantum metrology, it is asymmetry which is relevant, not entanglement.

Finally, the resource theory approach provides a framework for organizing and consolidating the results in a given field, thermodynamics being particularly in need of such a framework, as well as synthesizing new results. Indeed, studying the interconvertibility of finite resources leads to useful notions of free energy in that case, as shown in parallel to the present work in Ref. [7], and subsequently to a more detailed, quantitative treatment of the Second Law in Ref. [8]. Results similar to the former were also reported independently of [7] and the present work by Åberg [9] and later by Egloff et al. [10], who investigated the work extractable from finite resources. For a different approach in the same quantum setting as ours, but more along the lines of statistical mechanics, see Ref. [11].

Allowed operations and resource states.-We now define the restricted class of operations and the resource states more precisely. Given a quantum system with Hilbert space $\mathcal{H}$ and Hamiltonian $H$, the restricted operations are the completely positive trace-preserving maps $\mathcal{E}: \mathcal{L}(\mathcal{H}) \rightarrow \mathcal{L}(\mathcal{H})$ of the form

$$
\mathcal{E}(\rho)=\operatorname{Tr}_{\text {anc }}\left[V\left(\rho \otimes \bar{\gamma}_{T}\right) V^{\dagger}\right],
$$

where $\bar{\gamma}_{T}$ is the thermal (Gibbs) state of an arbitrary ancillary system with Hamiltonian $\bar{H}$ at temperature $T, V$ is an arbitrary unitary operation on the joint system which commutes with the total Hamiltonian: $[V, H \otimes I+I \otimes$ $\bar{H}]=0$. Observe that $\mathcal{E}\left(\gamma_{T}\right)=\gamma_{T}$, where $\gamma_{T}$ is the Gibbs state at temperature $T$ associated with $H$. Any other state $\rho \neq \gamma_{T}$ is a resource state. While, here, we consider the case that input and output systems and their Hamiltonians are identical, this framework can be easily extended to the more general case, as done by Janzing et al. [6].

The allowed operations are particularly relevant for thermodynamics because they cannot, on their own, be used to do work. Moreover, it is not too difficult to see that various different kinds of $T$-athermal states can be used, via the restricted class of operations, to do work. For thermal states at a temperature $T^{\prime}$ distinct from $T$ (hence $T$ athermal), work can be drawn using a heat engine, as we now effectively have two heat baths at temperatures $T$ and $T^{\prime}$; for pure states within a degenerate energy eigenspace, work can be drawn using a Szilard engine [12]; for pure energy eigenstates, work can be drawn directly by an energy-conserving unitary. One is led to expect that work can be extracted from any $T$-athermal state. We shall show that asymptotically this is, indeed, the case.

It is important to note the differences between the resource theory framework and the more usual approaches to thermodynamics. Chiefly, all sources and sinks of energy and entropy must be explicitly accounted for: only energy and entropy-neutral operations on the system and thermal reservoir are allowed, rather than specific energyor entropy-changing operations more common in an opensystem approach. All interactions between the system and reservoir are due to the unitary $V$ and not an interaction term in the total Hamiltonian. Moreover, no attempt is made a priori to restrict the allowed operations to be physically realistic; indeed, we assume the experimenter has complete control over $V$. This ensures that the restrictions we find are truly fundamental, though ultimately the operations needed to establish our main result are mappings between macroscopic observables and do not require fine-grained, microscopic control. These apparent differences notwithstanding, we show in the Supplemental Material [13] that a number of different classes of operations for thermodynamics are, in fact, equivalent.

Resource interconvertibility and free energy.-A central question in any resource theory is that of resource interconversion: Which resources can be transformed into which others, and how easily? Generally, there exists a quasiorder of resources: We say $A \geq B$ if resource $A$ can be transformed into $B$ using the allowed operations. Functions which respect this quasiorder are known as resource monotones. For instance, the relative entropy of entanglement is a well-known resource monotone relative to local operations and classical communication [1].

Here, we are interested in determining the optimal rate $R(A \rightarrow B)$ at which resource $A$ can generate resource $B$, in the limit of an infinite supply of $A$, that is, the largest $R$ such that $A^{\otimes n} \geq B^{\otimes n R}$ for $n \rightarrow \infty$. A simple argument, going back to Carnot [14], implies that if the transformation is reversible in the sense that $R(B \rightarrow A)=R(A \rightarrow B)^{-1}$, then the rate at which two resources can be reversibly interconverted must achieve the optimal rate. Otherwise, it would be possible to generate arbitrary amounts of a resource state from a small number via cyclic transformations to and from another resource state.

That reversible interconversion is optimal (when possible) gives a simple means of characterizing the interconversion rate by using a "standard" reference resource. Consider a transformation from $A$ to $B$ which proceeds via the standard resource $C: A \rightarrow C \rightarrow B$. Following this with $B \rightarrow A$ must give a combined transformation of unit rate, again to avoid the possibility of spontaneously generating resources. Composing the rates, we have $R(A \rightarrow C) R(C \rightarrow B) R(B \rightarrow A)=1$, or 


$$
R(A \rightarrow B)=\frac{R(A \rightarrow C)}{R(B \rightarrow C)},
$$

using the fact that $R(A \rightarrow B) R(B \rightarrow A)=1$. With this framework, we need only define the relative entropy $D\left(\rho \| \gamma_{T}\right)=\operatorname{Tr}\left[\rho\left(\ln \rho-\ln \gamma_{T}\right)\right]$ to state the main result of this Letter.

Theorem 1.-Using thermal operations at background temperature $T$, asymptotic interconversion at nonvanishing rate is possible between all states $\rho$ and $\sigma$ of a system with Hamiltonian $H$. For $\gamma_{T}$ the Gibbs state of temperature $T$ associated with $H$, the optimal rate is given by

$$
R(\rho \rightarrow \sigma)=\frac{D\left(\rho \| \gamma_{T}\right)}{D\left(\sigma \| \gamma_{T}\right)} .
$$

Simple calculation reveals that $D\left(\rho \| \gamma_{T}\right)=\beta F_{\beta}(\rho)-$ $\beta F_{\beta}\left(\gamma_{T}\right)$, where $F_{\beta}(\rho) \equiv\langle H\rangle_{\rho}-k_{B} T S(\rho)$ is the free energy and $S(\rho)=-\operatorname{Tr}[\rho \ln \rho]$ the von Neumann entropy. Thus, the free energy directly determines the optimal rate of resource interconversion in our resource theory.

To prove the result we shall employ the connection to free energy by constructing protocols for both distillation of resource states into a standard state and formation of resource states from standard states. The standard state is chosen to have energy but no entropy, so as to represent available work.

Before doing so, it is enlightening to note that, assuming reversible interconversion is possible, Eq. (3) follows easily from Ref. [15], Theorem 1, and Ref. [16], Theorem 4. This result states that any asymptotically continuous resource monotone $f$ determines the interconversion rate via its regularization $f^{\infty}(\rho)=\lim _{n \rightarrow \infty}(1 / n) f\left(\rho^{\otimes n}\right)$ as $R(\rho \rightarrow$ $\sigma)=f^{\infty}(\rho) / f^{\infty}(\sigma)$, provided the latter is nonzero and finite.

Here, $f(\rho)=D\left(\rho \| \gamma_{T}\right)$ is a $T$-athermality monotone (i.e., for all $T$-thermal operations $\mathcal{E}, D\left[\mathcal{E}(\rho) \| \gamma_{T}\right] \leq$ $\left.D\left(\rho \| \gamma_{T}\right)\right)$ by contractivity of the relative entropy under quantum operations and the fact that $\mathcal{E}\left(\gamma_{T}\right)=\gamma_{T}$. Its regularization is nonzero and finite since $f^{\infty}(\rho)=f(\rho)$, which follows from the additivity of the relative entropy and the fact the thermal state of $n$ identical systems is just $n$ copies of the thermal state of one system. Finally, asymptotic continuity follows from extensivity of energy by using Proposition 2 of Ref. [17]; we leave the simple derivation of this to the Supplemental Material [13].

Extensivity is crucial to the conclusion. For instance, $\tilde{f}(\rho)=D\left(\gamma_{T} \| \rho\right)$ (note the reversed order of $\rho$ and $\gamma_{T}$ ) is also a $T$-athermality monotone, but does not lead to the interconversion rate; the extensivity argument fails and $\tilde{f}$ is not asymptotically continuous. Nonetheless, $\tilde{f}(\rho)$ plays an important role in determining the resource requirements for creating low-temperature states [6].

Distillation and formation protocols.-In order to establish Theorem 1, let us now turn to the distillation and formation protocols. For purposes of exposition, we specialize to the case of resources having just two nondegenerate energy levels, call them $|0\rangle$ and $|1\rangle$, i.e., qubits. This, nevertheless, captures the essential aspects of the problem. First, we consider the distillation and formation of quasiclassical resources $\rho$, meaning $[\rho, H]=0$ and take up the case of nonstationary resources afterwards. In what follows, we sketch the steps required to complete the proof and leave the somewhat cumbersome mathematical details to the Supplemental Material [13].

Both distillation and formation protocols must satisfy three requirements, up to error terms smaller than $O(n)$ : (1) energy conservation, (2) unitarity, and (3) equality of input and output dimensions. Without loss of generality, we may take the total Hamiltonian to be $H=E_{0} \sum_{i}|1\rangle_{i}\langle 1|$ for some energy $E_{0}$, where the sum runs over all the qubits.

We begin the distillation protocol with $\ell$ copies of the Gibbs state $\gamma_{T}$ of $H$ and $n$ copies of the resource $\rho$, where $\rho=(1-p)|0\rangle\langle 0|+p| 1\rangle\langle 1|$ for arbitrary $0 \leq p \leq 1$ and $\gamma_{T}=(1-q)|0\rangle\langle 0|+q| 1\rangle\langle 1|$ for $q=e^{-\beta E_{0}} /\left(1+e^{-\beta E_{0}}\right)$. The aim is to effect a transformation of the form $\gamma_{T}^{\otimes \ell} \otimes$ $\rho^{\otimes n} \rightarrow \sigma^{(k)} \otimes|1\rangle\left\langle\left. 1\right|^{\otimes m}\right.$ by an energy-conserving unitary, such that $m$ is as large as possible. The resulting exhaust state $\sigma$ of $k$ systems is arbitrary, though as an aside we show that the optimality of the protocol implies that it has near-Gibbs form in the Supplemental Material [13]. We denote by $R=(m / n)$ the rate of distillation and $\epsilon=(n / l)$ the ratio between initial resource states and Gibbs states. The Gibbs states are free, so we allow $\epsilon \rightarrow 0$ as $n \rightarrow \infty$.

We now use the fact that for large $n, \rho^{\otimes n}$ consists of mixtures of basis states corresponding to length $n$ binary strings with roughly $n p 1 \mathrm{~s}$. The number $t$ of $1 \mathrm{~s}$ in a string is known as its type, and more concretely we have that, up to an error which vanishes as $n \rightarrow \infty$,

$$
\rho^{\otimes n} \approx \sum_{t} p_{t} P_{t} .
$$

Here the $t$ summation runs over strongly typical types, the types for which $t=n p \pm O(\sqrt{n})$, and $P_{t}$ denotes the projector onto the type $t$. See Ref. [18], Sec. 2, for more details. An entirely similar statement holds for $\gamma_{T}^{\otimes \ell}$. For simplicity, we shall first pretend that both $\gamma_{T}^{\otimes \ell}$ and $\rho^{\otimes n}$ consist of a single type and subsequently show how to extend the argument to all strongly typical types.

We begin with a single composite type, a concatenation of a type coming from the resource state and one from the reservoir state. This corresponds to a uniform mixture of strings of length $n+\ell$, each of which consists of two substrings: the first having $\ell q$ 1s and the second $n p 1 \mathrm{~s}$. There are roughly $e^{l h(q)} \times e^{n h(p)}$ such strings, where $h(p)=-p \ln p-(1-p) \ln (1-p)$ is the binary entropy, expressed in nats.

Now consider a transformation which maps these strings to new strings having at least $m 1 \mathrm{~s}$ in the rightmost positions, 


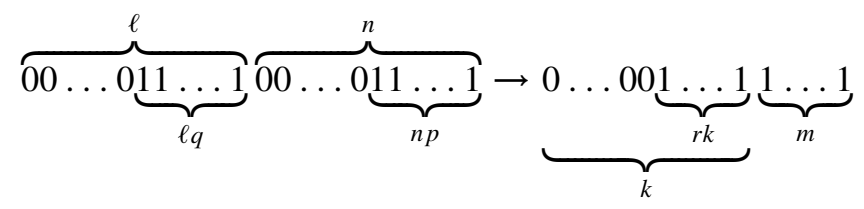

where $k=\ell+n-m$ expresses conservation of dimension, and $r$ and $m$ are to be determined. Conservation of energy requires that the number of $1 \mathrm{~s}$ is conserved, hence, $\ell q+n p=r k+m$. Unitarity requires that there are at least as many strings of length $k$ with $r k 1 \mathrm{~s}$ as the number of initial strings: $e^{k h(r)} \geq e^{\ell h(q)+n h(p)}$. Roughly speaking, this is conservation of entropy. Using these three conditions, we find that the transformation is possible for any $R$ such that

$$
h(q)+\epsilon h(p) \leq(1+\epsilon-R \epsilon) h\left(\frac{q+\epsilon p-\epsilon R}{1+\epsilon-\epsilon R}\right) .
$$

We now expand this with respect to $\epsilon$ to first order and let $\epsilon \rightarrow 0$. This means the heat reservoir is much larger than the resource systems. As a result, we obtain that the following rate can be achieved:

$$
R=\frac{h(q)-h(p)+\beta(p-q)}{h(q)+\beta(1-q)}=\frac{D\left(\rho \| \gamma_{T}\right)}{D\left(|1\rangle\langle 1| \| \gamma_{T}\right)},
$$

establishing one direction necessary for Theorem 1.

In the above argument, we worked with a single composite type, whereas in actuality, the initial state is a mixture of these. Thus, we apply the protocol separately to each composite type, assuming the number $m$ of output excited states to be the same for all input types, with $m$ fitted to the composite type containing the fewest strings (i.e., the one consisting of strings with $\ell q-O(\sqrt{\ell})+$ $n p-O(\sqrt{n}) 1$ 's). To proceed as above, we need to ensure that any variations from the above conditions are small relative to $n$. Thus, we need to simultaneously fulfill $\sqrt{\ell} \ll$ $m=R n$, in order for $R$ from (6) to be achievable, and $\ell \gg n$, in order for $\epsilon \rightarrow 0$. Choosing $\ell=(R n)^{3 / 2}$, therefore, ensures that our estimate (6) will be accurate in the limit $n \rightarrow \infty$ [19].

The formation protocol is similar to the distillation protocol and is again based on considering type transformations satisfying the three requirements of energy conservation, unitarity, and dimension conservation. The major difference is that, whereas the ideal distillation output is simply the fixed-type state $|1\rangle^{\otimes m}$, the ideal formation output must recreate a good approximation to the probabilistic mixture of type classes found in $\rho^{\otimes n}$.

We construct the formation protocol in three stages. The first two are similar to the distillation protocol. In the first, a given type class of the Gibbs state together with the standard resource is transformed into a desired type class of the target resource $\rho^{\otimes n}$. In the second, the transformation is extended to all the strongly typical types of the Gibbs state. Finally, in the third step, an additional number of Gibbs states are used to probabilistically select which type class of the target should be output, in order to recreate the appropriate distribution over types of the target state. In principle, this step is irreversible, but since the number of type classes grows only polynomially with $n$, the number of extra resources required for the third step of the formation protocol vanishes in the $n \rightarrow \infty$ limit. The similarity of the first two steps with the distillation protocol then ensures that the formation protocol achieves the inverse rate.

Distillation for arbitrary resource states is related to that of stationary states, and we can recycle part of the previous distillation protocol. Suppose the resource state has the diagonal form $\rho=p\left|\phi_{1}\right\rangle\left\langle\phi_{1}|+(1-p)| \phi_{2}\right\rangle\left\langle\phi_{2}\right|$, for arbitrary orthogonal states $\left|\phi_{k}\right\rangle$, implying an average energy of $\langle E\rangle=\left(p\left|\left\langle\phi_{1} \mid 1\right\rangle\right|^{2}+(1-p)\left|\left\langle\phi_{2} \mid 1\right\rangle\right|^{2}\right) E_{0}$. In $n$ instances of $\rho$ the total energy will overwhelmingly likely be $n\langle E\rangle \pm O(\sqrt{n})$. Now, imagine projecting the resource state onto the various energy subspaces, destroying any coherence between them. Just as in (4), $\rho^{\otimes n}$ is supported almost entirely on its typical subspace, whose size is not larger than $e^{n S(\rho)+O(\sqrt{n})}$. Thus, the state support in every energy subspace is at most this large.

Now we may imagine applying the same scheme as in the previous distillation protocol, creating as many copies of $|1\rangle$ as possible. The three conditions now become $k=$ $\ell+n-m, \quad \ell q E_{0}+n\langle E\rangle=r k E_{0}+m E_{0}, \quad$ and $e^{k h(r)} \geq$ $e^{\ell h(q)+n S(\rho)}$. An entirely similar derivation leads again to the distillation rate found in (6). Finally, since the distillation operations commute with the Hamiltonian, they commute with the projection onto energy subspaces. Thus, we may, instead, imagine that this projection is performed after the distillation step. Such a projection has no effect on the work systems, while the form of the exhaust state is irrelevant, and therefore, we may dispense with the projection step altogether.

The formation of arbitrary resource states is more complicated than their distillation. Strictly speaking, the desired transformation is impossible, since the inputs are states diagonal in the energy basis and the allowed transformations cannot change this fact. However, to create the appropriate coherences between energy subspaces it suffices to use a small additional resource in the form of a superposition over energy eigenstates.

In particular, a system in a superposition of energy levels acts as a reference system which lifts the superselection rule of energy conservation, as in Refs. [20,21], allowing one to create arbitrary coherences over energy levels on the system. However, since $\rho^{\otimes n}$ is almost entirely supported on energy levels in the range $n\langle E\rangle \pm O(\sqrt{n})$, the formation process requires only a reference system made from order $\sqrt{n}$ qubits. The extra resource of the reference system is, thus, of a size sublinear in $n$ and does not affect the rate calculations. This creates an interesting asymmetry between distillation and formation, akin to a similar phenomenon in the resource theory of entanglement, where 
distillation of entangled states does not require any communication, but formation requires an amount sublinear in the number of inputs $n$.

Conclusions.-We have shown that well-known results from thermodynamics can be derived quite naturally within the framework of the resource theory of energypreserving transformations and auxiliary thermal states. We should emphasize that although the procedures we have described for the conversion of resource states may seem quite unnatural from a physical point of view, their use is to establish the "in principle" interconversion rate given in Theorem 1. Any more realistic reversible transformation such as, for instance, the Hamiltonian method of Ref. [22] or, for quasiclassical resources, the sequential protocol of Ref. [23] which was developed subsequently to the results presented here, will necessarily extract the same amount of work.

We thank Jochen Rau and Dominik Janzing for helpful conversations. J.M.R. acknowledges support from the Center for Advanced Security Research Darmstadt (CASED). R.W.S. acknowledges support from the Government of Canada through NSERC and the Province of Ontario through MRI. M. H. is grateful for the support of the Foundation for Polish Science TEAM project cofinanced by the EU European Regional Development Fund. Part of this work was done at the National Quantum Information Centre of Gdansk. The authors acknowledge the hospitality of the Institute Mittag Leffler within the program Quantum Information Science (2010), where part of this work was done.

*Corresponding author. renes@phys.ethz.ch

[1] R. Horodecki, P. Horodecki, M. Horodecki, and K. Horodecki, Rev. Mod. Phys. 81, 865 (2009).

[2] D. Janzing and T. Beth, IEEE Trans. Inf. Theory 49, 230 (2003).

[3] G. Gour and R. W. Spekkens, New J. Phys. 10, 033023 (2008).
[4] I. Marvian and R. W. Spekkens, New J. Phys. 15, 033001 (2013).

[5] M. Horodecki, P. Horodecki, and J. Oppenheim, Phys. Rev. A 67, 062104 (2003).

[6] D. Janzing, P. Wocjan, R. Zeier, R. Geiss, and T. Beth, Int. J. Theor. Phys. 39, 2717 (2000).

[7] M. Horodecki and J. Oppenheim, Nat. Commun. 4, 2059 (2013).

[8] F. G. S. L. Brandão, M. Horodecki, N.H. Y. Ng, J. Oppenheim, and S. Wehner, arXiv:1305.5278.

[9] J. Ảberg, Nat. Commun. 4, 1925 (2013).

[10] D. Egloff, O.C. O. Dahlsten, R. Renner, and V. Vedral, arXiv:1207.0434.

[11] B. Schumacher and M. Westmoreland, Quantum Processes Systems, and Information (Cambridge University Press, Cambridge, England, 2010).

[12] J. Oppenheim, M. Horodecki, P. Horodecki, and R. Horodecki, Phys. Rev. Lett. 89, 180402 (2002).

[13] See Supplemental Material at http://link.aps.org/ supplemental/10.1103/PhysRevLett.111.250404 for more precise proofs of Theorem 1 and statements made in the main text.

[14] E. Fermi, Thermodynamics (Courier Dover, New York, 1956).

[15] M. Horodecki, J. Oppenheim, and R. Horodecki, Phys. Rev. Lett. 89, 240403 (2002).

[16] G. Gour, I. Marvian, and R. W. Spekkens, Phys. Rev. A 80, 012307 (2009).

[17] K. Horodecki, M. Horodecki, P. Horodecki, and J. Oppenheim, Phys. Rev. Lett. 94, 200501 (2005).

[18] I. Csiszár and J. Körner, Information Theory: Coding Theorems for Discrete Memoryless Systems (Cambridge University Press, Cambridge, England, 2011), 2nd ed.

[19] Additionally, the number of strings of a given type $n p$ is given by poly $(n) e^{n h(p)}$, not simply $e^{n h x(p)}$. However, in the limit $n \rightarrow \infty$, the polynomial factor is again not relevant in our estimates.

[20] Y. Aharonov and L. Susskind, Phys. Rev. 155, 1428 (1967).

[21] S. D. Bartlett, T. Rudolph, and R. W. Spekkens, Rev. Mod. Phys. 79, 555 (2007).

[22] R. Alicki, M. Horodecki, P. Horodecki, and R. Horodecki, Open Syst. Inf. Dyn. 11, 205 (2004).

[23] P. Skrzypczyk, A. J. Short, and S. Popescu, arXiv:1302.2811. 